\title{
Structured Frames by Weil Entourages
}

In honour of Professor Bernhard Banaschewski on the occasion of his 70th birthday

\author{
JORGE PICADO \\ Departamento de Matemática, Universidade de Coimbra, Apartado 3008, 3000 Coimbra, Portugal \\ e-mail: picado@mat.uc.pt
}

(Received: 18 October 1996; accepted: 11 April 1998)

Abstract. In the framework of pointfree topology, we discuss the rôle of Weil entourages in the study of structures such as uniformities, quasi-uniformities, nearnesses, quasi-nearnesses, proximities and infinitesimal relations.

Mathematics Subject Classifications (2000): 06D20, 54E05, 54E15, 54E17.

Key words: frame, Weil entourage, uniform frame, localic group, quasi-uniform frame, nearness, quasi-nearness, proximity, infinitesimal relation.

In the same way as the notion of Boolean algebra appears as an abstraction of the power set $\mathcal{P}(X)$ of a set $X$, the notion of frame arises as an abstraction from the topology $\mathcal{T}$ of a topological space $(X, \mathcal{T})$ : a frame is a complete lattice $L$ satisfying the distributive law $x \wedge \bigvee S=\bigvee\{x \wedge s \mid s \in S\}$ for all $x \in L$ and $S \subseteq L$. A frame homomorphism is a map between frames which preserves finitary meets (including the unit 1) and arbitrary joins (including the zero 0). A standard reference for frames is Johnstone [18].

Pointfree topology deals with the category Frm of frames and frame homomorphisms (or, depending on the point of view, with its dual category of locales; in this 'localic' point of view one thinks of locales as generalized spaces; technically, however, we will work in Frm. Therefore, for example, statements on products of generalized spaces will appear as statements on coproducts of frames).

In topology, metrics, uniformities, nearnesses, proximities and such like structures are defined on a set and determine a topology on that set. In pointfree topology, "the specified object is the 'topology', that is, a frame; and consequently, metrics, uniformities, and nearnesses must appear as additional structures on a frame" [2].

The study of structured frames started in the early 1970's with Isbell [15], where uniformities on frames were introduced as the exact translation into frame terms of Tukey's approach [25] to uniform spaces via covers, and continued, in more 
detail, with Pultr [23, 24] and Frith [11]. Quasi-uniformities, which are uniformities without symmetry, were introduced by Frith [11] in terms of conjugate cover pairs of biframes (the translation into frame terms of the approach of Gantner and Steinlage [13] to quasi-uniform spaces via covers). Nearness, which is uniformity without the star-refinement condition, was introduced more recently by Banaschewski and Pultr [4], and in [5] and [6] Dube studied several properties of nearness frames.

As in the case of spaces, there are other ways of describing (quasi-)uniformities, such as the entourage (quasi-)uniformities of Fletcher and Hunsaker [9] and the Weil (quasi-)uniformities of Picado [20, 21]. The various descriptions are known to be equivalent.

The purpose of this article is to outline some results of my $\mathrm{Ph} . \mathrm{D}$. thesis, written under the supervision of Professors B. Banaschewski and M. Sobral, which I believe establish the naturality of an entourage approach to several frame structures (uniformities, quasi-uniformities, nearnesses, proximities etc.). The point that I wish to make here is that the notion of Weil entourage introduced in [20] is the exact translation into frame terms of Weil's classical notion of entourage and that it provides us with the appropriate environment to get frame extensions of spatial results envolving entourages, and to formulate them in a very similar way to the corresponding classical results. After a brief summary, in a preliminary section, on the needed tools, I describe some results of [21] which emphasize this point.

I would like to express my gratitude to Professor B. Banaschewski for his invaluable suggestions and comments.

\section{Preliminaries}

For subsets $A, B, \ldots$ and elements $x, y, \ldots$ of a frame $L$, let us recall the following notation and terminology:

$A \leq B$ if each member of $A$ is below some member of $B$

$$
\begin{aligned}
& \text { st }(x, A):=\bigvee\{a \in A \mid a \wedge x \neq 0\}, \quad A^{*}:=\{s t(x, A) \mid x \in A\}, \\
& A \wedge B:=\{a \wedge b \mid a \in A, b \in B\} .
\end{aligned}
$$

Further, $A$ is called a cover of $L$ if $\bigvee A=1$, and $\operatorname{Cov}(L)$ denotes the set of all covers of $L$, taken as quasi-ordered by the above relation $\leq$. Next, for any $\mathcal{A} \subseteq \operatorname{Cov}(L), y \stackrel{\mathcal{A}}{\triangleleft} x$ means that $s t(y, A) \leq x$ for some $A \in \mathcal{A}$, and $\mathcal{A}$ is called admissible whenever $x=\bigvee\{y \in L \mid y \stackrel{\mathcal{A}}{\triangleleft} x\}$ for all $x \in L$. Then, a nearness on $L$ is an admissible filter $U$ in $\operatorname{Cov}(L)$. In particular, $U$ is called a uniformity if it satisfies the refinement condition, that is, for each $U \in \mathcal{U}$, there exists $V \in \mathcal{U}$ such that $V^{*} \leq U$. A nearness frame (resp., uniform frame) is a frame together with a specified nearness (resp., uniformity). For nearness 
frames $(L, \mathcal{U})$ and $\left(L^{\prime}, \mathcal{U}^{\prime}\right)$, a frame map $f: L \rightarrow L^{\prime}$ is uniform if $f[U] \in \mathcal{U}^{\prime}$ for any $U \in \mathcal{U}$. Further, Nfrm (resp., Ufrm) will be the category of nearness frames and uniform homomorphisms (resp., uniform frames and uniform homomorphisms).

For the following, recall that a subset $A$ of a poset $(X, \leq)$ is said to be a down-set if $A=\downarrow A$ where $\downarrow A$ denotes the set $\{x \in X \mid x \leq a$ for some $a \in A\}$.

Let $L$ be a frame. Recall (cf., e.g., [18]) that the coproduct of the frame $L$ by itself

$$
L \stackrel{u_{1}^{L}}{\longrightarrow} L \oplus L \stackrel{u_{2}^{L}}{\longleftarrow} L
$$

can be constructed as follows:

Take the Cartesian product $L \times L$ with the usual order. A down-set $A$ of $L \times L$ is a $C$-ideal if $(\{x\} \times S \subseteq A \Rightarrow(x, \bigvee S) \in A)$ and $(S \times\{y\} \subseteq A \Rightarrow(\bigvee S, y) \in A)$. Put $L \oplus L$ as the frame of all $C$-ideals of $L \times L$. Observe that the case $S=\emptyset$ implies that every $C$-ideal contains the set $\mathbb{O}:=\downarrow\{(1,0)\} \cup \downarrow\{(0,1)\}$. Obviously, each $\downarrow\{(x, y)\} \cup \mathbb{O}$ is a $C$-ideal. It is denoted by $x \oplus y$. Finally put $u_{1}^{L}(x)=x \oplus 1$ and $u_{2}^{L}(y)=1 \oplus y$.

The following clear facts are useful:

- For every $A \in L \oplus L, A=\bigvee\{x \oplus y \mid(x, y) \in A\}$ and so every element of $L \oplus L$ is join-generated by some family of elements $x \oplus y$;

- $\mathbb{O} \neq x \oplus y \subseteq z \oplus w$ implies $x \leq z$ and $y \leq w$.

For any frame homomorphism $f: L \rightarrow M$, we write $f \oplus f: L \oplus L \rightarrow M \oplus M$ for the frame homomorphism given by $(f \oplus f) \cdot u_{i}^{L}=u_{i}^{M} \cdot f(i=1,2)$. Obviously, $(f \oplus f)\left(\bigvee_{\gamma}\left(x_{\gamma} \oplus y_{\gamma}\right)\right)=\bigvee_{\gamma}\left(f\left(x_{\gamma}\right) \oplus f\left(y_{\gamma}\right)\right)$.

Given $A, B$ in the lattice $\mathcal{D}(L \times L)$ of all down-sets of $L \times L$ we denote by $k(A)$ the $C$-ideal generated by $A$ and by $A \circ B$ the $C$-ideal generated by $\{(x, y) \in L \times L$ | $\exists z \in L \backslash\{0\}:(x, z) \in A,(z, y) \in B\}$, that is, $\bigvee\{x \oplus y \mid \exists z \in L \backslash\{0\}:(x, z) \in$ $A,(z, y) \in B\}$. The following technical lemma, proved in [20] (Lemma 3.1), will play a crucial rôle in our approach:

LEMMA A. For any $A, B \in \mathscr{D}(L \times L), k(A) \circ k(B)=A \circ B$.

The importance of this result relies in the fact that it allows us to work with composition of frame entourages in a way very similar to the spatial one. This can be observed, for example, in the proof of Proposition 1.4 below. 


\section{Weil Structures on Frames}

\section{UNIFORMITIES}

Uniform spaces were introduced by Weil in terms of the basic notion of entourage. An entourage of a set $X$ is a subset $E$ of $X \times X$ for which the diagonal $\Delta_{X}: X \rightarrow$ $X \times X$ factorizes through the inclusion $E \hookrightarrow X \times X$ :

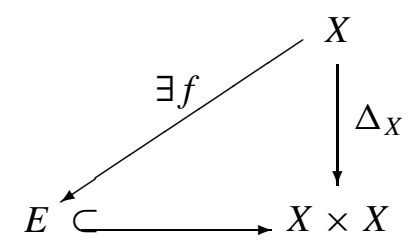

This motivates the study, on a frame $L$, of the elements $E$ of the frame coproduct $L \oplus L$ for which the codiagonal $\nabla_{L}: L \oplus L \rightarrow L$ factorizes through the open sublocale $L \oplus L \rightarrow \downarrow\{E\}$ of $L \oplus L$ :

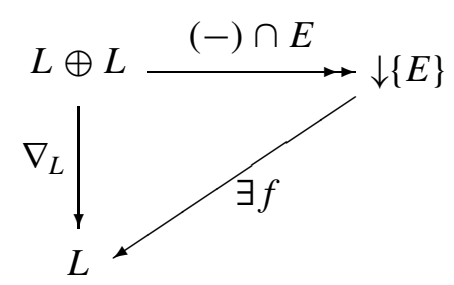

(here, $(-) \cap E(F)=E \cap F$, for every $F \in L \oplus L$ and $\nabla_{L}$ is the unique morphism such that $\left.\nabla_{L} \cdot u_{1}^{L}=1_{L}=\nabla_{L} \cdot u_{2}^{L}\right)$. We call them Weil entourages of $L$. Evidently, $E \in L \oplus L$ is a Weil entourage if and only if $\nabla_{L}(E)=1$. Since $\nabla_{L}$ is defined by

$$
E=\bigvee_{(x, y) \in E}(x \oplus y) \longmapsto \bigvee_{(x, y) \in E}(x \wedge y),
$$

an element $E$ of $L \oplus L$ is a Weil entourage if and only if $\bigvee_{(x, x) \in E} x=1$ or, in other words, if and only if there exists a cover $U$ of $L$ such that $\bigvee_{x \in U}(x \oplus x) \subseteq E$. This shows a perfect analogy between our notion of frame entourage (introduced in [20]) and the notion of entourage for sets and justifies the designation of Weil entourage for these frame entourages.

The collection $W E n t(L)$ of all Weil entourages of $L$ may be partially ordered by inclusion. This is a partially ordered set with finitary meets (including a unit $1=L \oplus L)$.

We define the composition of Weil entourages as follows:

$$
E \circ F:=\bigvee\{x \oplus y \mid \exists z \in L \backslash\{0\}:(x, z) \in E,(z, y) \in F\}
$$

For the basic properties of the operation o see [21]. 
The inverse of a Weil entourage $E$ has the natural definition $E^{-1}=\{(y, x) \mid$ $(x, y) \in E\}$. We also consider a new partial order in $L$, induced by a family $\mathcal{E}$ of Weil entourages:

$$
\begin{aligned}
& y \stackrel{\mathcal{E}}{\triangleleft} x(\text { read ' } y \text { is } \mathcal{E} \text {-strongly below } x \text { ') if there is } \\
& \quad E \in \mathcal{E} \text { such that } E \circ(y \oplus y) \subseteq x \oplus x .
\end{aligned}
$$

Of course, when $\mathscr{E}$ is symmetric (that is, $E \in \mathcal{E}$ implies $E^{-1} \in \mathcal{E}$ ) this is equivalent to saying that

$$
\text { there is } E \in \mathcal{E} \text { such that }(y \oplus y) \circ E \subseteq x \oplus x .
$$

DEFINITION 1.3. Let $L$ be a frame and let $\mathcal{E}$ be a nonempty filter of $(\operatorname{WEnt}(L), \subseteq)$. Consider the following axioms:

(W1) [Admissibility Axiom] For any $x \in L, x=\bigvee\{y \in L \mid y \stackrel{\bar{\varepsilon}}{\triangleleft} x\}$ (where $\overline{\mathcal{E}}$ denotes the filter of $(\operatorname{WEnt}(L), \subseteq)$ generated by $\left.\mathscr{E} \cup\left\{E^{-1} \mid E \in \mathcal{E}\right\}\right)$;

(W2) [Symmetry Axiom] For any $E \in \mathcal{E}, E^{-1} \in \mathcal{E}$;

(W3) [Refinement Axiom] For each $E \in \mathcal{E}$ there exists $F \in \mathcal{E}$ such that $F \circ F \subseteq E$.

The family $\mathcal{E}$ is called a Weil nearness on $L$ if it satisfies (W1) and (W2) and it is called a Weil uniformity on $L$ if it fulfils (W1), (W2) and (W3). The pair $(L, \mathcal{E})$ is called a Weil nearness frame (resp., Weil uniform frame) if $\varepsilon$ is a Weil nearness (resp., Weil uniformity) on L. A Weil nearness base (resp., Weil uniformity base) is just a filter base of some Weil nearness (resp., Weil uniformity).

If $(L, \mathscr{E})$ and $\left(L^{\prime}, \mathcal{E}^{\prime}\right)$ are Weil nearness frames, a frame homomorphism $f$ : $L \rightarrow L^{\prime}$ is called a Weil homomorphism from $(L, \mathcal{E})$ to $\left(L^{\prime}, \mathcal{E}^{\prime}\right)$ if $(f \oplus f)(E) \in \mathcal{E}^{\prime}$ whenever $E \in \mathcal{E}$.

We shall denote by WNFrm (resp., WUFrm) the category of Weil nearness frames and Weil homomorphisms (resp., Weil uniform frames and Weil homomorphisms).

We proceed to give examples of Weil uniformities which illustrate the similarities with the corresponding spatial cases.

EXAMPLES. (a) Let $(L, d)$ be a metric frame [24]. For any real $\epsilon>0$ let $E_{\epsilon}=$ $\bigvee\{x \oplus x \mid d(x)<\epsilon\}$. The family $\left(E_{\epsilon}\right)_{\epsilon>0}$ is a base for a Weil uniformity on $L$.

(b) Consider a localic group [16] (a cogroup in the category of frames), that is, a frame $L$ endowed with a multiplication $\mu: L \rightarrow L \oplus L$, an inverse $t: L \rightarrow L$ and a unit point $\varepsilon: L \rightarrow \mathbf{2}$ ( 2 denotes the two-element lattice) satisfying the identities

$$
\begin{aligned}
& \left(\mu \oplus 1_{L}\right) \cdot \mu=\left(1_{L} \oplus \mu\right) \cdot \mu, \\
& \left(\varepsilon \oplus 1_{L}\right) \cdot \mu=1_{L}=\left(1_{L} \oplus \varepsilon\right) \cdot \mu
\end{aligned}
$$


and

$\nabla_{L} \cdot\left(\imath \oplus 1_{L}\right) \cdot \mu=\nabla_{L} \cdot\left(1_{L} \oplus \imath\right) \cdot \mu=\sigma \cdot \varepsilon$,

where $\sigma$ is the morphism $\mathbf{2} \rightarrow L$. Note that the usual properties for groups

$$
\begin{aligned}
& \varepsilon \cdot \imath=\varepsilon, \\
& \imath \cdot \imath=1_{L}
\end{aligned}
$$

and

$$
\mu \cdot \imath=\tau \cdot(l \oplus \imath) \cdot \mu,
$$

where $\tau$ is the unique map from $L \oplus L$ to $L \oplus L$ satisfying $\tau \cdot u_{1}^{L}=u_{2}^{L}$ and $\tau \cdot u_{L}^{2}=u_{L}^{1}$, are also valid here.

These groups have Weil uniformities that arise in a similar way as in the spatial setting of topological groups. For any $x \in L$ such that $\varepsilon(x)=1$ put

$$
E_{x}^{l}:=\left(1_{L} \oplus \imath\right)(\mu(x)) \quad \text { and } \quad E_{x}^{r}:=\left(\imath \oplus 1_{L}\right)(\mu(x)) .
$$

PROPOSITION 1.4. $\mathcal{E}^{l}:=\left\{E_{x}^{l} \mid x \in L, \varepsilon(x)=1\right\}$ and $\mathbb{E}^{r}:=\left\{E_{x}^{r} \mid x \in\right.$ $L, \varepsilon(x)=1\}$ are bases for Weil uniformities.

Proof. We only show that $\mathcal{E}^{r}$ is a Weil uniformity base. The proof for $\mathcal{E}^{l}$ is similar.

Each $E_{x}^{r}$ is a Weil entourage because $\nabla_{L}\left(E_{x}^{r}\right)=\nabla_{L} \cdot\left(\imath \oplus 1_{L}\right) \cdot \mu(x)=\varepsilon(x)=1$.

Obviously, $E_{x}^{r} \cap E_{y}^{r}=E_{x \wedge y}^{r}$. Since $\varepsilon(x \wedge y)=1$ whenever $\varepsilon(x)=\varepsilon(y)=1$, $\mathcal{E}^{r}$ is a filter base of $(\operatorname{WEnt}(L), \subseteq)$.

The symmetry is a consequence of the fact that, for every $x,\left(E_{x}^{r}\right)^{-1}=E_{l(x)}^{r}$ which we prove next. Put $\mu(x)=\bigvee_{\gamma \in \Gamma}\left(x_{\gamma} \oplus y_{\gamma}\right)$. Then $E_{x}^{r}=\bigvee_{\gamma \in \Gamma}\left(l\left(x_{\gamma}\right) \oplus y_{\gamma}\right)$. On the other hand, since $\mu \cdot l=\tau \cdot(l \oplus l) \cdot \mu$, we have $\mu(l(x))=\bigvee_{\gamma \in \Gamma}\left(l\left(y_{\gamma}\right) \oplus l\left(x_{\gamma}\right)\right)$ and, therefore, $E_{l(x)}^{r}=\bigvee_{\gamma \in \Gamma}\left(y_{\gamma} \oplus \imath\left(x_{\gamma}\right)\right)$.

Now, consider $E_{x}^{r}$ with $\varepsilon(x)=1$. We have $\varepsilon=1_{\mathbf{2}} \oplus \varepsilon=\left(1_{\mathbf{2}} \oplus \varepsilon\right) \cdot\left(\varepsilon \oplus 1_{L}\right) \cdot \mu=$ $(\varepsilon \oplus \varepsilon) \cdot \mu$. Thus $(\varepsilon \oplus \varepsilon) \cdot \mu(x)=1$, that is, $\bigvee\{\varepsilon(a) \oplus \varepsilon(b) \mid(a, b) \in \mu(x)\}=1$. Therefore there is some $(a, b) \in \mu(x)$ with $\varepsilon(a)=\varepsilon(b)=1$. Also $(a \wedge b, a \wedge b) \in$ $\mu(x)$ and $\varepsilon(a \wedge b)=1$. Denote $a \wedge b$ by $y$. We claim that $E_{y}^{r} \circ E_{y}^{r} \subseteq E_{x}^{r}$. In fact, $E_{y}^{r} \circ E_{y}^{r}$ is the Weil entourage

$$
\begin{aligned}
& \left(\bigvee_{(a, b) \in \mu(y)}(l(a) \oplus b)\right) \circ\left(\bigvee_{(a, b) \in \mu(y)}(l(a) \oplus b)\right) \\
& =\left(\bigcup_{(a, b) \in \mu(y)}(l(a) \oplus b)\right) \circ\left(\bigcup_{(a, b) \in \mu(y)}(l(a) \oplus b)\right),
\end{aligned}
$$

by Lemma A. Take $(l(a), b)$ with $(a, b) \in \mu(y)$ and $(l(c), d)$ with $(c, d) \in \mu(y)$ such that $b \wedge l(c) \neq 0$. From the inclusion $y \oplus y \subseteq \mu(x)$ it follows that

$$
\mu(y) \oplus \mu(y) \subseteq(\mu \oplus \mu)(\mu(x))=\left(1_{L} \oplus \mu \oplus 1_{L}\right) \cdot\left(\mu \oplus 1_{L}\right) \cdot(\mu(x)) .
$$


Therefore $a \oplus b \oplus c \oplus d \subseteq\left(1_{L} \oplus \mu \oplus 1_{L}\right) \cdot\left(\mu \oplus 1_{L}\right) \cdot(\mu(x))$. Applying $1_{L} \oplus \nabla_{L}$. $\left(1_{L} \oplus \imath\right) \oplus 1_{L}$ to both sides we get

$$
a \oplus(b \wedge l(c)) \oplus d \subseteq\left(1_{L} \oplus \sigma \oplus 1_{L}\right) \cdot(\mu(x))=\bigvee_{(a, b) \in \mu(x)}\left(a \oplus 1_{L} \oplus b\right)
$$

Since $b \wedge l(c) \neq 0$, then $(a, d) \in \mu(x)$ and $(l(a), d) \in E_{x}^{r}$. Hence $E_{y}^{r} \circ E_{y}^{r} \subseteq E_{x}^{r}$.

Finally, let us check the admissibility condition (W1). From the identity $\left(1_{L} \oplus\right.$ $\varepsilon) \cdot \mu=1_{L}$, it follows that, for every $x \in L$,

$$
\begin{aligned}
x & =\bigvee\{a \oplus \varepsilon(b) \mid a \oplus b \subseteq \mu(x)\} \\
& =\bigvee\{a \in L \mid \exists b \in L: \varepsilon(b)=1 \text { and } a \oplus b \subseteq \mu(x)\},
\end{aligned}
$$

whence it remains to show that $a$ is $\mathcal{E}^{r}$-strongly below $x$ whenever there is some $b \in L$ satisfying $\varepsilon(b)=1$ and $a \oplus b \subseteq \mu(x)$. In order to conclude this, it suffices to show that, for any symmetric $F \in \mathcal{E}^{r}$ such that $F^{2} \subseteq E_{b}^{r}, F \circ(a \oplus a) \subseteq x \oplus x$. So, assume $(\alpha, \beta) \in F,(\beta, \gamma) \leq(a, a)$ with $\alpha, \beta, \gamma \neq 0$. We need to show that both $\alpha$ and $\gamma$ are below $x$. Clearly $\gamma \leq a \leq x$. Let us show that also $\alpha \leq x$. Of course, $(\alpha, \beta) \in F^{2}$ and, by the symmetry of $F,(\beta, \alpha) \in F^{2}$, which forces $(\alpha \vee \beta, \alpha \vee \beta) \in F^{2} \subseteq E_{b}^{r}$, as $(\alpha, \alpha)$ and $(\beta, \beta)$ also belong to $F^{2}$. Moreover $(\alpha \vee \beta) \wedge a \neq 0$. Thus $l(\alpha \vee \beta) \oplus(\alpha \vee \beta) \subseteq \mu(b)$. On the other hand, $a \oplus b \subseteq$ $\mu(x)$. Consequently, $a \oplus b \oplus a \subseteq\left(\mu \oplus 1_{L}\right)(x \oplus a)$ which, in turn, implies that $a \oplus \mu(b) \oplus a \subseteq\left(1_{L} \oplus \mu \oplus 1_{L}\right) \cdot\left(\mu \oplus 1_{L}\right)(x \oplus a)$. Thus, we have $a \oplus l(\alpha \vee \beta) \oplus a \subseteq$ $\left(1_{L} \oplus \mu \oplus 1_{L}\right) \cdot\left(\mu \oplus 1_{L}\right)(x \oplus a)$. By the associativity of $\mu,\left(1_{L} \oplus \mu \oplus 1_{L}\right) \cdot\left(\mu \oplus 1_{L}\right)=$ $\left(\left(1_{L} \oplus \mu\right) \cdot \mu\right) \oplus 1_{L}=\left(\left(\mu \oplus 1_{L}\right) \cdot \mu\right) \oplus 1_{L}=\left(\mu \oplus 1_{L} \oplus 1_{L}\right) \cdot\left(\mu \oplus 1_{L}\right)$. Thus, $a \oplus l(\alpha \vee \beta) \oplus(\alpha \vee \beta) \oplus a \subseteq\left(\mu \oplus 1_{L} \oplus 1_{L}\right) \cdot\left(\mu \oplus 1_{L}\right)(x \oplus a)$. Applying $\left(\nabla_{L} \cdot\left(1_{L} \oplus \imath\right)\right) \oplus 1_{L} \oplus 1_{L}$ to both sides we get $(a \wedge(\alpha \vee \beta)) \oplus(\alpha \vee \beta) \oplus a \subseteq$ $\left(\sigma \cdot \varepsilon \oplus 1_{L} \oplus 1_{L}\right) \cdot\left(\mu \oplus 1_{L}\right)(x \oplus a)=\left(\left(\sigma \cdot \varepsilon \oplus 1_{L}\right) \cdot \mu \oplus 1_{L}\right)(x \oplus a)=(((\sigma \oplus$ $\left.\left.\left.1_{L}\right) \cdot\left(\varepsilon \oplus 1_{L}\right) \cdot \mu\right) \oplus 1_{L}\right)(x \oplus a)=\left(\sigma \oplus 1_{L} \oplus 1_{L}\right)(x \oplus a)$. But $\left(\sigma \oplus 1_{L}\right)(x)=1 \oplus x$ so $(a \wedge(\alpha \vee \beta)) \oplus(\alpha \vee \beta) \oplus a \subseteq 1 \oplus x \oplus a$. Since $a \wedge(\alpha \vee \beta) \neq 0$, we finally obtain $(\alpha \vee \beta) \oplus a \subseteq x \oplus a$. In conclusion, $\alpha \leq \alpha \vee \beta \leq x$.

We point out that, as for topological groups, the map $t$ is a Weil uniform frame isomorphism between the two structures: indeed, $\imath$ is a frame isomorphism and, for any $x \in L,(\imath \oplus \imath)\left(E_{x}^{r}\right)=(\imath \oplus \imath)\left(\imath \oplus 1_{L}\right)(\mu(x))=\left(1_{L} \oplus \imath\right)(\mu(x))=E_{x}^{l}$.

(c) Our approach via Weil entourages may also be useful to prove (or disprove) frame counterparts of spatial results envolving entourages. For an example in this direction see [21], where it is proved that whenever two uniformities with countable bases on a frame determine the same Samuel compactification they are equal. This is the localic version of a theorem of Efremovic of 1952 [8] (cf. Theorem 12.18 and Corollary 12.19 of [19]).

Weil entourages and covers interact in a very easy way: for any Weil entourage $E$ the set $\{x \in L \mid x \oplus x \subseteq E\}$ is a cover of $L$ and, conversely, for any cover $U$ 
of $L, \bigvee_{x \in U}(x \oplus x)$ is a Weil entourage of $L$. Moreover, for any nearness frame $(L, \mathcal{U}),\left\{\bigvee_{x \in U}(x \oplus x) \mid U \in \mathcal{U}\right\}$ forms a base for a Weil nearness $\Psi(\mathcal{U})$ on $L$ and, conversely, for any Weil nearness frame $(L, \mathcal{E}),\{\{x \in L \mid x \oplus x \subseteq E\} \mid E \in \mathcal{E}\}$ forms a base for a nearness $\Phi(\mathcal{E})$ on $L$.

The correspondences $(L, \mathcal{U}) \stackrel{\Psi}{\longmapsto}(L, \Psi(\mathcal{U}))$ and $(L, \mathcal{E}) \stackrel{\Phi}{\longmapsto}(L, \Phi(\mathcal{E}))$ constitute a Galois connection between the partially ordered sets (by inclusion) of, respectively, nearnesses and Weil nearnesses on $L: 1 \leq \Psi \Phi$ and $\Phi \Psi \leq 1$. This Galois connection induces a 'maximal' isomorphism between the partially ordered set of Weil nearnesses $\mathscr{E}$ on $L$ satisfying the condition

$$
\forall E \in \mathcal{E} \bigvee_{(x, x) \in E}(x \oplus x) \in \mathcal{E}
$$

and the partially ordered set of nearnesses $\mathcal{U}$ on $L$ satisfying the condition

$$
\forall U \in U \exists V \in U:\left(x \oplus x \subseteq \bigvee_{x \in V}(x \oplus x) \Rightarrow \exists u \in U: x \leq u\right)
$$

For the proofs and further details, the reader is referred to [21]. This extends the result of [20] that asserts the isomorphism between WUFrm and UFrm; in fact, in particular, this gives a bijection for uniformities since it can be readily seen that the refinement conditions in the definitions of Weil uniform frame and uniform frame are stronger than the corresponding conditions 1.3 and 1.4 above. However, I am not able to conclude whether WNFrm and NFrm are isomorphic. This is still an open question.

\section{QUASI-UNIFORMITIES AND QUASI-NEARNESSES}

One of the advantages of entourage-like theories is that the symmetry is explicit and so the corresponding nonsymmetric versions are evident and pleasantly manageable. By dropping the symmetry in Definitions 1.3 we have the notions of Weil quasi-nearness frame and Weil quasi-uniform frame.

With the lack of symmetry the equivalence between conditions 1.1 and 1.2 is no longer valid; whence, in the place of $\stackrel{\mathcal{E}}{\triangleleft}$ we have two order relations

$$
\begin{aligned}
& y \stackrel{\mathcal{E}}{\triangleleft}_{1} x \equiv E \circ(y \oplus y) \subseteq x \oplus x, \quad \text { for some } E \in \mathcal{E}, \\
& y \stackrel{\mathcal{\&}}{\triangleleft}_{2} x \equiv(y \oplus y) \circ E \subseteq x \oplus x, \quad \text { for some } E \in \mathcal{E},
\end{aligned}
$$

which in turn, lead to two subframes of $L$,

$$
\begin{aligned}
& L_{1}:=\left\{x \in L \mid x=\bigvee\left\{y \in L \mid y \dot{\vee}_{1} x\right\}\right\} \text { and } \\
& L_{2}:=\left\{x \in L \mid x=\bigvee\left\{y \in L \mid y \triangleleft_{2} x\right\}\right\}
\end{aligned}
$$


(for spaces this corresponds to the two topologies defined by the quasi-uniformity).

Notice that the condition (W1) of admissibility says that the triple $\left(L, L_{1}, L_{2}\right)$ is a biframe. This allows us to establish a Galois connection between Weil entourages and conjugate cover pairs of biframes:

Recall that a biframe [3] is a triple $B=\left(B_{0}, B_{1}, B_{2}\right)$ in which $B_{0}$ is a frame and $B_{1}, B_{2}$ are subframes of $B_{0}$ such that each $x \in B_{0}$ is the join of finite meets from $B_{1} \cup B_{2}$.

Let $B=\left(B_{0}, B_{1}, B_{2}\right)$ be a biframe. The following definitions and notations are transcribed from [11]. A subset $U$ of $B_{1} \times B_{2}$ is a conjugate cover pair (or just conjugate cover $)$ of $B$ provided that $\left\{u_{1} \wedge u_{2} \mid\left(u_{1}, u_{2}\right) \in U\right\}$ is a cover of $B_{0}$. A conjugate cover $U$ is strong if, for every $\left(u_{1}, u_{2}\right) \in U, u_{1} \wedge u_{2} \neq 0$ whenever $u_{1} \vee u_{2} \neq 0$. Let $U$ and $V$ be conjugate covers. Then $U \leq V$ provided that, for each $\left(u_{1}, u_{2}\right) \in U$, there is $\left(v_{1}, v_{2}\right) \in V$ with $u_{1} \leq v_{1}$ and $u_{2} \leq v_{2}$. Furthermore, $U \wedge V$ denotes the conjugate cover $\left\{\left(u_{1} \wedge v_{1}, u_{2} \wedge v_{2}\right) \mid\left(u_{1}, u_{2}\right) \in U,\left(v_{1}, v_{2}\right) \in V\right\}$ and $U^{*}$ stands for the conjugate cover $\left\{\left(s t_{1}\left(u_{1}, U\right), s t_{2}\left(u_{2}, U\right)\right) \mid\left(u_{1}, u_{2}\right) \in U\right\}$, where $s t_{1}(x, U):=\bigvee\left\{u_{1} \mid\left(u_{1}, u_{2}\right) \in U, u_{2} \wedge x \neq 0\right\}$ and $s t_{2}(x, U):=\bigvee\left\{u_{2} \mid\right.$ $\left.\left(u_{1}, u_{2}\right) \in U, u_{1} \wedge x \neq 0\right\}$ for any $x \in B_{0}$.

Let $B=\left(B_{0}, B_{1}, B_{2}\right)$ be a biframe and let $U$ be a collection of conjugate covers. Then $\mathcal{U}$ is a quasi-uniformity for the biframe $B$ and $(B, \mathcal{U})$ is a quasi-uniform frame provided that:

(i) The family of strong members of $U$ is a filter base for $U$ with respect to $\leq$;

(ii) For each $U \in U$ there exists $V \in \mathcal{U}$ such that $V^{*} \leq U$;

(iii) For each $x \in B_{i}(i \in\{1,2\}), x=\bigvee\left\{y \in B_{i} \mid y \triangleleft_{i}^{u} x\right\}$, where $y \triangleleft_{i}^{u} x$ means that there is $U \in \mathcal{U}$ such that $s t_{i}(y, U) \leq x$.

Let $(B, \mathcal{U})$ and $\left(B^{\prime}, \mathcal{U}^{\prime}\right)$ be quasi-uniform frames. A uniform homomorphism $f:(B, \mathcal{U}) \rightarrow\left(B^{\prime}, \mathcal{U}^{\prime}\right)$ is a biframe map $f: B \rightarrow B^{\prime}$ such that, for every $U \in \mathcal{U}$, $f[U]:=\left\{\left(f\left(u_{1}\right), f\left(u_{2}\right)\right) \mid\left(u_{1}, u_{2}\right) \in U\right\} \in \mathcal{U}^{\prime}$. The category of quasi-uniform frames and uniform homomorphisms will be denoted by QUFrm.

The relationship between Weil entourages and conjugate covers is not so evident as the one between Weil entourages and covers. For any conjugate cover $U$ of a biframe $\left(B_{0}, B_{1}, B_{2}\right), \bigvee\left\{u_{1} \oplus u_{2} \mid\left(u_{1}, u_{2}\right) \in U\right\}$ (meant in $B_{0} \oplus B_{0}$ ) is a Weil entourage of $B_{0}$. Conversely, starting with a Weil entourage $E$ of a quasi-nearness frame $\mathrm{L}$, we may consider the biframe $\left(L, L_{1}, L_{2}\right)$ and the pairs $\left(s t_{1}(x, E), s t_{2}(x, E)\right)$ for $(x, x) \in E$, where $s t_{1}(x, E):=\bigvee\{y \in L \mid(y, z) \in$ $E, z \wedge x \neq 0\}$ and $s t_{2}(x, E):=\bigvee\{y \in L \mid(z, y) \in E, z \wedge x \neq 0\}$. However there is a problem: these pairs may not belong to $L_{1} \times L_{2}$. It may be solved by introducing two interior operators in $L \oplus L$ in the following manner: For every $I, J \in L \oplus L$, we define $I \stackrel{\mathcal{E}}{\complement}_{1} J$ by $E \circ I \subseteq J$ for some $E \in \mathcal{E}$, and similarly we define $I \stackrel{\mathcal{E}}{\sqsubseteq}_{2} J$ by $I \circ E \subseteq J$ for some $E \in \mathcal{E}$. Further, for each $i \in\{1,2\}$, let $\operatorname{int}_{i}(E):=\bigvee\{I \in$ $\left.L \oplus L \mid I \stackrel{\mathcal{E}}{E}_{i} E\right\}$. Then $\left\{\left(s t_{1}\left(x, \operatorname{int}_{1}(E)\right), \operatorname{st}_{2}\left(x, \operatorname{int}_{2}(E)\right)\right) \mid x \oplus x \subseteq E\right\}$ is already a 
conjugate cover of $\left(L, L_{1}, L_{2}\right)$. These correspondences define a Galois connection which gives an isomorphism for quasi-uniformities (see [22] for the details). For quasi-nearnesses the question of knowing whether the entourage approach and the cover approach are equivalent is still open.

Notice that Weil quasi-uniformities are defined on a frame; do not require as the (biframe) quasi-uniformities - prior knowledge of the underlying biframe. The analogy with the spatial case is now evident. This clarifies the discussion in D. Doitchinov, "Some reflections on quasi-uniform frames", Topology with Applications (Szekszárd, Hungary, 1993), Bolyai Math. Soc., Math. Studies 4, 1995, pp. 151-158.

A natural question concerning Weil (quasi-)nearness frames is: which is the right spatial concept in analogy with the chosen frame concept, that is, which spaces correspond to them by an 'open'/'spectrum' adjunction?

The answer (see [21]) gives us the categories WNear (of 'Weil nearness spaces') and WQNear (of 'Weil quasi-nearness spaces'). We concluded in Chapter IV of [21] that WNear is an interesting category containing the categories of symmetric topological spaces and continuous maps, uniform spaces and uniformly continuous maps, and proximal spaces and proximal maps, in a nice way: they are either bireflective or bicoreflective full subcategories of WNear. However the category Top of topological spaces is not a subcategory of WNear. Other useful topological structures, namely the nonsymmetric ones of quasi-uniform spaces and quasi-proximal spaces are also not embeddable in WNear.

Nevertheless, in the realm of those 'Weil quasi-nearness spaces' mentioned above, it is possible to consider all those spaces of topological and uniform nature. It turns out that the category of Weil quasi-nearness spaces contains all the nonsymmetric categories referred to above as well as the category Top as nicely embedded full subcategories and so it is a unified theory of (nonsymmetric) topology and uniformity. The corresponding covering results were explained in the thesis [11].

\section{PROXIMITIES}

Let us now see how it goes with proximities. We recall that a binary relation $\ll$ on a frame $L$ is a strong inclusion [1] if the following axioms are satisfied for $x, y, z, w$ in $L$ :

(P1) $x \leq y \ll z \leq w$ implies $x \ll w$;

(P2) $\ll$ is a sublattice of $L \times L$;

(P3) $x \ll y$ implies $x \prec y$ (where $x \prec y$ means that $x \wedge z=0$ and $y \vee z=1$ for some $z \in L$, alternatively expressed as $y \vee x^{*}=1$ with the pseudocomplement $\left.x^{*}=\bigvee\{z \in L \mid x \wedge z=0\}\right)$;

(P4) If $x \ll y$ then $x \ll z \ll y$ for some $z \in L$; 
(P5) $x \ll y$ implies $y^{*} \ll x^{*}$;

(P6) For each $x \in L, x=\bigvee\{y \in L \mid y \ll x\}$.

Note that, in the presence of (P1), (P2) means: $0 \ll 0,1 \ll 1, x \ll y$ and $z \ll y$ imply $x \vee z \ll y$, and $x \ll y$ and $x \ll z$ imply $x \ll y \wedge z$.

The pair $(L, \ll)$ is called a proximal frame if $\ll$ is a strong inclusion on $L$. A frame map between proximal frames is said to be proximal if it preserves the strong inclusion. We denote by PFrm the category of proximal frames and proximal frame homomorphisms.

REMARKS 3.1. (a) The basic properties of Weil uniformities imply that, for any Weil uniform frame $(L, \mathcal{E}),(L, \triangleleft)$ is a proximal frame.

(b) When $x \vee y=1$, the $C$-ideal $(x \oplus x) \vee(y \oplus y)$ is a Weil entourage of $L$ (the converse is also true; for the proof the reader is referred to [21]). Thus, in any proximal frame $(L, \ll)$, since $\ll$ is stronger than $\prec, E_{x, y}:=\left(x^{*} \oplus x^{*}\right) \vee(y \oplus y)$ is a Weil entourage whenever $x \ll y$.

The entourages $E_{x, y}$ are very important. Indeed, they enable us to formulate the concept of proximity in terms of Weil entourages:

PROPOSITION 3.2. Let $(L, \ll)$ be a proximal frame. Then $\left\{E_{x, y} \mid x, y \in L, x \ll\right.$ $y$ \} is a subbase for a Weil uniformity $\mathcal{E}(\ll)$ on L. Moreover, the proximity $\stackrel{\mathcal{E}(\ll)}{\triangleleft}$ induced by $\mathcal{E}(\ll)$ coincides with $\ll$.

Proof. It is an immediate consequence of Lemma IV.6.7 of [21] (which has a rather technical and long proof that we omit here). Indeed, this lemma affirms that, in any proximal frame $(L, \ll), x \ll y$ whenever $\bigcap_{i=1}^{n} E_{x_{i}, y_{i}} \subseteq E_{x, y}$ and $x_{i} \ll y_{i}$ for every $i \in\{1, \ldots, n\}$.

The following proposition is also obvious:

PROPOSITION 3.3. If $(L, \mathcal{E})$ is a Weil uniform frame satisfying

$$
\text { (W4) } \forall E \in \mathcal{E} \exists x_{1}, y_{1}, \ldots, x_{n}, y_{n} \in L:\left(\bigcap_{i=1}^{n} E_{x_{i}, y_{i}} \subseteq E \text { and } \bigcap_{i=1}^{n} E_{x_{i}, y_{i}} \in \mathcal{E}\right) \text {, }
$$

the Weil uniformity $\mathcal{E}(\stackrel{\mathcal{E}}{\triangleleft})$ induced by $\stackrel{\mathcal{E}}{\triangleleft}$ coincides with $\mathcal{E}$.

Let us denote by WUFrm(W4) the full subcategory of WUFrm of all Weil uniform frames satisfying (W4). This is a bicoreflective subcategory of WUFrm.

The preceding propositions give us a characterization of frame proximities in terms of Weil entourages:

THEOREM 3.4. The categories PFrm and $\mathrm{WUFrm}_{(\mathrm{W} 4)}$ are isomorphic. 
Proof. According to Remark 3.1(a) and Propositions 3.2 and 3.3 it remains to show that a frame map $f: L_{1} \rightarrow L_{2}$ between two proximal frames $\left(L_{1}, \ll_{1}\right)$ and $\left(L_{2}, \ll_{2}\right)$ is a proximal frame homomorphism if and only if it is a Weil uniform homomorphism from $\left(L_{1}, \mathcal{E}\left(\ll_{1}\right)\right)$ to $\left(L_{2}, \mathcal{E}\left(\ll_{2}\right)\right)$. So, let $f$ be a proximal frame homomorphism. For any $E \in \mathcal{E}\left(\ll_{1}\right)$ we may write $\bigcap_{i=1}^{n} E_{x_{i}, y_{i}} \subseteq E$, where $x_{i} \ll_{1}$ $y_{i}$ for every $i \in\{1, \ldots, n\}$. Then

$$
\begin{aligned}
(f \oplus f)(E) & \supseteq \bigcap_{i=1}^{n}(f \oplus f)\left(E_{x_{i}, y_{i}}\right) \\
& =\bigcap_{i=1}^{n}\left(\left(f\left(x_{i}^{*}\right) \oplus f\left(x_{i}^{*}\right)\right) \vee\left(f\left(y_{i}\right) \oplus f\left(y_{i}\right)\right)\right) .
\end{aligned}
$$

Now consider, for each $i \in\{1, \ldots, n\}, z_{i}, w_{i} \in L$ such that $x_{i} \ll_{1} z_{i} \ll_{1} w_{i} \ll_{1} y_{i}$. There is a simple and well-known lemma (cf., for example, Lemma 2.8 of [11]), which affirms that, for any frame homomorphism $f, f(y)^{*} \leq f\left(x^{*}\right)$ whenever $x \prec y$. Applying this lemma, we obtain

$$
\begin{aligned}
& \left(f\left(x_{i}^{*}\right) \oplus f\left(x_{i}^{*}\right)\right) \vee\left(f\left(y_{i}\right) \oplus f\left(y_{i}\right)\right) \\
& \quad \supseteq\left(f\left(z_{i}\right)^{*} \oplus f\left(z_{i}\right)^{*}\right) \vee\left(f\left(w_{i}\right) \oplus f\left(w_{i}\right)\right)=E_{f\left(z_{i}\right), f\left(w_{i}\right)} .
\end{aligned}
$$

So $(f \oplus f)(E) \supseteq \bigcap_{i=1}^{n} E_{f\left(z_{i}\right), f\left(w_{i}\right)}$. Since, by hypothesis, $f\left(z_{i}\right) \ll_{2} f\left(w_{i}\right)$, then $(f \oplus f)(E) \in \mathcal{E}\left(\ll_{2}\right)$.

Conversely, if $x \ll_{1} y$ then $(f \oplus f)\left(E_{x, y}\right) \in \mathcal{E}\left(\ll_{2}\right)$. As $(f \oplus f)\left(E_{x, y}\right) \subseteq$ $E_{f(x), f(y)}$, the entourage $E_{f(x), f(y)}$ also belongs to $\mathscr{E}\left(\ll_{2}\right)$ and we may write $\bigcap_{i=1}^{n} E_{x_{i}, y_{i}} \subseteq E_{f(x), f(y)}$ for $x_{1}, y_{1}, \ldots, x_{n}, y_{n} \in L_{2}$ with $x_{i} \ll_{2} y_{i}$ for every $i \in\{1, \ldots, n\}$. It suffices now to recall again Lemma IV.6.7 of [21] to conclude that $f(x) \ll 2 f(y)$.

As it is well-known, the concepts of proximity and totally bounded uniformity are still equivalent for frames (the proof is due to Frith [12]). This motivates the following definition:

A Weil entourage $E$ is finite provided there exist elements $x_{1}, y_{1}, \ldots, x_{n}, y_{n}$ in $L$ such that $x_{i} \prec y_{i}$, for every $i \in\{1, \ldots, n\}$, and $\bigcap_{i=1}^{n} E_{x_{i}, y_{i}}=E$. By Remark 3.1(b), the conditions $x_{i} \prec y_{i}$ are redundant since each $E_{x_{i}, y_{i}}$ by containing $E$ is also a Weil entourage.

Now Theorem 3.4 affirms that

"The category PFrm is isomorphic to the full subcategory of WUFrm of Weil uniform frames with a base of finite entourages",

which is the entourage version of Frith's result.

When the frame $L$ is normal (i.e., $x \vee y=1$ implies there exist $u, v \in L$ such that $x \vee u=1=y \vee v$ and $u \wedge v=0$ ) our notion of finiteness may be stated in a way very likely to the spatial one: 
PROPOSITION 3.5. If $L$ is normal, the filter FWEnt $(L)$ of (WEnt $(L), \subseteq)$ generated by all finite Weil entourages coincides with the filter generated by

$$
\left\{\bigvee_{i=1}^{n}\left(x_{i} \oplus x_{i}\right) \mid n \in \mathbb{N}, x_{1}, \ldots, x_{n} \in L, \bigvee_{i=1}^{n} x_{i}=1\right\}
$$

Proof. Consider $E \in F W E n t(L)$. Then there are $x_{1}, y_{1}, \ldots, x_{n}, y_{n} \in L$ such that $x_{i} \prec y_{i}$ for every $i \in\{1, \ldots, n\}$ and $\bigcap_{i=1}^{n} E_{x_{i}, y_{i}} \subseteq E$. Since each $E_{x_{i}, y_{i}}$ belongs to the set

$$
\left\{\bigvee_{i=1}^{n}\left(x_{i} \oplus x_{i}\right) \mid n \in \mathbb{N}, x_{1}, \ldots, x_{n} \in L, \bigvee_{i=1}^{n} x_{i}=1\right\},
$$

$E$ belongs to the filter generated by it.

Conversely, consider $\bigvee_{i=1}^{n}\left(x_{i} \oplus x_{i}\right)$ with $\bigvee_{i=1}^{n} x_{i}=1$. From the normality of $L$ we may ensure the existence of $y_{1}, \ldots, y_{n} \in L$ such that $\bigvee_{i=1}^{n} y_{i}=1$ and $y_{i} \prec x_{i}$ for any $i \in\{1, \ldots, n\}$. Since $(L \oplus L, \cap, \bigvee)$ is a frame, one can easily prove, by induction on $n \geq 1$, that

$$
\bigcap_{i=1}^{n} E_{y_{i}, x_{i}}=\bigvee_{z_{1} \in\left\{y_{1}^{*}, x_{1}\right\}} \ldots \bigvee_{z_{n} \in\left\{y_{n}^{*}, x_{n}\right\}}\left(z_{1} \wedge \cdots \wedge z_{n} \oplus z_{1} \wedge \cdots \wedge z_{n}\right) .
$$

But $\left(y_{1}^{*} \wedge \cdots \wedge y_{n}^{*}\right) \oplus\left(y_{1}^{*} \wedge \cdots \wedge y_{n}^{*}\right)=\mathbb{O}$. Hence $\bigcap_{i=1}^{n} E_{y_{i}, x_{i}} \subseteq \bigvee_{i=1}^{n}\left(x_{i} \oplus x_{i}\right)$ and therefore $\bigvee_{i=1}^{n}\left(x_{i} \oplus x_{i}\right) \in \operatorname{FWEnt}(L)$.

REMARKS 3.6. (a) By dropping the symmetry of $\&$ in the definition of the objects of the category WUFrm ${ }_{(\mathrm{W} 4)}$ we obtain the theory of quasi-proximities in terms of Weil entourages. The isomorphism between WQUFrm and QUFrm yields an isomorphism between this category of 'quasi-proximal frames' and the covering one of Frith ([11], p. 68).

(b) The isomorphism between PFrm and WUFrm $\left(\mathrm{w}_{4}\right)$ suggested to us the notion of finite Weil entourage. It also justifies the fact that we name the Weil nearness frames satisfying (W4) as 'Weil contigual frames'. This is the analogous notion to the contigual frames of Dube [6] in the setting of nearness frames. One natural question is this: are these two categories equivalent? Another interesting problem is whether the corresponding category of 'Weil contigual spaces' (as a full subcategory of WNear) is equivalent to the category of contigual nearness spaces of Herrlich [14] and, consequently, equivalent to the classical category of contigual spaces and contigual maps in the sense of Ivanova and Ivanov [17].

In this paper we have tried to emphasize the point that our language of Weil entourages allows us to get frame versions of spatial results concerning entourages, and to formulate them in a very similar way to the corresponding classical results. We end it with another illustration of this. Although not entirely surprising, it is 
still noteworthy that Weil entourages also serve the same purpose for uniform-like structures in frames as entourages for spaces and that, after all, covers much wider ground, since there are structured frames which are not spatial.

\section{INFINITESIMAL RELATIONS}

The notion of proximity in classical topology was originaly introduced by Efremovič [7] in terms of the infinitesimal relation ' $A$ is near $B$ ' (usually denoted by $A \delta B$ [19]) for subsets $A$ and $B$ of any set. We conclude this article with a brief discussion of the corresponding axiomatization for frames.

PROPOSITION 4.1. If $(L, \ll)$ is a proximal frame, the binary relation on $L$ given by $x \delta y \equiv x \ll y^{*}$ satisfies the following properties:

(I1) $x \delta y$ implies $y \delta x$;

(I2) $x \delta(y \vee z)$ if and only if $x \delta y$ or $x \delta z$;

(I3) $x \delta y$ implies $x \neq 0$ and $y \neq 0$;

(I4) If $x \phi y$ then there is $z \in L$ such that $x \not z$ and $y \not z^{*}$;

(I5) $x^{*} \vee y^{*} \neq 1$ implies $x \delta y$;

(I6) For every $x \in L, x=\bigvee\left\{y \in L \mid y \leq x\right.$ and $\left.y \varnothing x^{*}\right\}$.

\section{Proof. Straightforward.}

Note that, since $x \wedge y \neq 0$ implies $x^{*} \vee y^{*} \neq 1$, (I5) says, in particular, that $x \delta y$ whenever $x \wedge y \neq 0$. When $L$ is Boolean the converse is also true and condition (I5) is equivalent to the condition $(x \wedge y \neq 0 \Rightarrow x \delta y)$.

We say that a binary relation $\delta$ satisfying all properties (I1)-(I6) of 4.1 is an infinitesimal relation and that, in this case, $(L, \delta)$ is an infinitesimal frame.

The correspondence of 4.1 is invertible for Boolean frames:

PROPOSITION 4.2. If $(L, \delta)$ is an infinitesimal frame, the binary relation « given by $x \ll y \equiv x \not y^{*}$ is a proximity on $L$ if and only if $L$ is Boolean.

Proof. If $\ll$ is a proximity then, for any $x \in L, x^{* *}=\bigvee\left\{y \in L \mid y \ll x^{* *}\right\}$. But $y \ll x^{* *} \Leftrightarrow y \varnothing x^{* * *} \Leftrightarrow y \ll x$. Consequently, $x^{* *}=\bigvee\{y \in L \mid y \ll x\}=x$ and $L$ is Boolean.

Since in any Boolean frame the DeMorgan law $\left(x_{1} \wedge x_{2}\right)^{*}=x_{1}^{*} \vee x_{2}^{*}$ also holds, the proof that $\ll$ is a proximity when $L$ is Boolean follows immediately from the properties of $\delta$.

These two results show that the description of proximities in terms of infinitesimal relations is also valid for Boolean frames. 
It is a well-known result of proximal spaces that, given a uniform space $(X, \mathscr{E})$, there is a way of defining an infinitesimal relation on $X$ by $A \delta B$ if and only if one of the following three equivalent conditions holds:

(i) For every $E \in \mathcal{E},(A \times B) \cap E \neq \emptyset$;

(ii) For every $E \in \mathscr{E}$ there is $a \in A$ such that $E[a] \cap B \neq \emptyset$;

(iii) For every $E \in \mathscr{E}$ there is $b \in B$ such that $A \cap E[b] \neq \emptyset$.

In frames, we have a similar situation. In fact, the next proposition, together with Remark 3.1(a) and Proposition 4.1, says that any Weil uniformity $\&$ on $L$ induces an infinitesimal relation $\delta$ on $L$ by $x \delta y$ if and only if one of the following three equivalent conditions is satisfied:

(i) For every $E \in \mathcal{E},(x \oplus y) \cap E \neq \mathbb{O}$;

(ii) For every $E \in \mathcal{E}$ there exists $x^{\prime} \in \downarrow\{x\} \backslash\{0\}$ such that $E\left[x^{\prime}\right] \wedge y \neq 0$;

(iii) For every $E \in \mathscr{E}$ there exists $y^{\prime} \in \downarrow\{y\} \backslash\{0\}$ such that $x \wedge E\left[y^{\prime}\right] \neq 0$.

PROPOSITION 4.3. Let $\&$ be a Weil nearness on L and let $x, y \in$ L. Denoting by $E[x](E \in \mathcal{E})$ the element $\bigvee\{y \in L \mid(x, y) \in E\}$, the following assertions are equivalent:

(i) $x \stackrel{\mathcal{E}}{\triangleleft} y^{*}$;

(ii) $(x \oplus y) \cap E=\mathbb{O}$ for some $E \in \mathcal{E}$;

(iii) There is $E \in \mathcal{E}$ such that, for every nonzero $x^{\prime} \in \downarrow\{x\}, E\left[x^{\prime}\right] \wedge y=0$;

(iv) There is $E \in \mathcal{E}$ such that, for every nonzero $y^{\prime} \in \downarrow\{y\}, x \wedge E\left[y^{\prime}\right]=0$.

Proof. The equivalence between (i) and (ii) is a consequence of the equivalence between $(x \oplus y) \cap E^{-1}=\mathbb{O}$ and $E \circ(x \oplus x) \subseteq y^{*} \oplus y^{*}$, that we prove next. So, let $(x \oplus y) \cap E^{-1}=\mathbb{O}$ and consider $(a, b) \in E$ and $(b, c) \in x \oplus x$ with $a, b, c \neq 0$. Then $(b, a \wedge y) \in E^{-1} \cap(x \oplus y)=\mathbb{O}$ and $a \wedge y=0$, that is, $a \leq y^{*}$. On the other hand $x \leq y^{*}$, i.e., $x \wedge y=0$. In fact, $x \wedge y=\bigvee\{x \wedge y \wedge a \mid(a, a) \in E\}$ and, for any $(a, a) \in E,(x \wedge y \wedge a, x \wedge y \wedge a) \in(x \oplus y) \cap E=\mathbb{O}$. Thus $c \leq x \leq y^{*}$ and $(a, c) \in y^{*} \oplus y^{*}$. The reverse implication is also clear.

The equivalence (ii) $\Leftrightarrow$ (iii) is obvious. By symmetry, (ii) is then also equivalent to (iv).

\section{Acknowledgement}

Financial assistance from the Centro de Matemática da Universidade de Coimbra, the JNICT project PraxisXXI/ESCoLa and the Organising Committee of BBFest96 is gratefully acknowledged. 


\section{References}

1. Banaschewski, B.: Compactifications of frames, Math. Nachr. 149 (1990), 105-115.

2. Banaschewski, B.: Completion in Pointfree Topology, Lecture Notes in Math. and Appl. Math. 2/96, University of Cape Town, 1996.

3. Banaschewski, B., Brümmer, G. C. L. and Hardie, K. A.: Biframes and bispaces, Quaestiones Math. 6 (1983), 13-25.

4. Banaschewski, B. and Pultr, A.: Cauchy points of uniform and nearness frames, Quaestiones Math. 19 (1996), 101-127.

5. Dube, T. A.: Structures in frames, Ph.D. Thesis, University of Durban, Westville, 1993.

6. Dube, T. A.: The Tamano-Dowker type theorems for nearness frames, J. Pure Appl. Algebra 99 (1995), 1-7.

7. Efremovič, V. A.: Infinitesimal spaces, Dokl. Akad. Nauk 76 (1951), 341-343.

8. Efremovič, V. A.: The geometry of proximity I, Mat. Sb. N.S. 31 (1952), 189-200.

9. Fletcher, P. and Hunsaker, W.: Entourage uniformities for frames, Monatsh. Math. 112 (1991), 271-279.

10. Fletcher, P., Hunsaker, W. and Lindgren, W.: Frame quasi-uniformities, Monatsh. Math. 117 (1994), 223-236.

11. Frith, J. L.: Structured frames, Ph.D. Thesis, University of Cape Town, 1987.

12. Frith, J. L.: The category of uniform frames, Cahiers Topologie Géom. Différentielle Catég. 31 (1990), 305-313.

13. Gantner, T. E. and Steinlage, R. C.: Characterizations of quasi-uniformities, J. London Math. Soc. (2) 5 (1972), 48-52.

14. Herrlich, H.: Topological structures, in Topological Structures I, Math. Centre Tracts 52, 1974, pp. 59-122.

15. Isbell, J.: Atomless parts of spaces, Math. Scand. 31 (1972), 5-32.

16. Isbell, J., Kř́žz, I., Pultr, A. and Rosícki, J.: Remarks on localic groups, in F. Borceux (ed.), Categorical Algebra and its Applications (Proc. Int. Conf. Louvain-La-Neuve 1987), Lecture Notes in Math. 1348, pp. 154-172.

17. Ivanova, V. M. and Ivanov, A. A.: Contiguity spaces and bicompact extensions, Izv. Akad. Nauk. SSSR 23 (1959), 613-634.

18. Johnstone, P. T.: Stone Spaces, Cambridge Stud. Adv. Math. 3, Cambridge University Press, Cambridge, 1982.

19. Naimpally, S. A. and Warrack, B. D.: Proximity Spaces, Cambridge University Press, Cambridge, 1970.

20. Picado, J.: Weil uniformities for frames, Comment. Math. Univ. Carolin. 36 (1995), 357-370.

21. Picado, J.: Weil entourages in pointfree topology, Ph.D. Thesis, University of Coimbra, 1995.

22. Picado, J.: Frame quasi-uniformities by entourages, in Festschrift for GCL Brümmer on his 60th birthday, Department of Mathematics, University of Cape Town, 1998.

23. Pultr, A.: Pointless uniformities I. Complete regularity, Comment. Math. Univ. Carolin. 25 (1984), 91-104.

24. Pultr, A.: Pointless uniformities II. (Dia)metrization, Comment. Math. Univ. Carolin. 25 (1984), 105-120.

25. Tukey, J. W.: Convergence and Uniformity in Topology, Ann. Math. Stud. 2, Princeton University Press, 1940.

26. Weil, A.: Sur les espaces à structure uniforme et sur la topologie générale, Publications de l'Institute Mathématique de l'Université de Strasbourg, Hermann, Paris, 1938. 